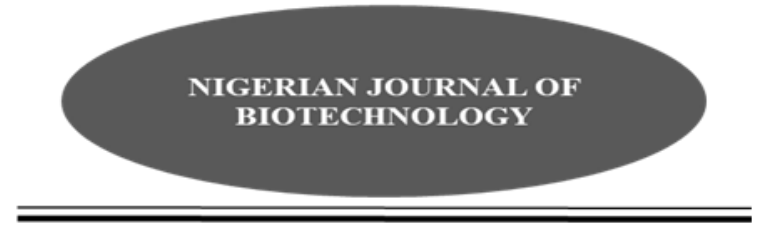

\title{
Comparative Fatty Acid Profiling of Klebsiella pneumoniae and Rhodococcus rhodochrous Isolated from Spoilt Paints by Gas Chromatography
}

\author{
Obidi, O. F. \\ Department of Microbiology, University of Lagos, Akoka, Lagos, Nigeria.
}

\begin{abstract}
The use of fatty acids to study the differences in un-related microbes is limited. This study analyzes the fatty acids produced by two unrelated microorganisms: Klebsiella pneumoniae (Gram-negative, aerobic, non-endospore forming, usually encapsulated rod-shaped bacteria of the family Enterobacteriaceae) and Rhodococcus rhodochrous (metabolically versatile, non-spore-forming, non-motile actinomycete) isolated from spoilt paints. Fatty acids produced by the organisms were analyzed using an efficient MIDI-Sherlock gas chromatography method . K. pneumoniae was characterized by a high content of straight chain, branched chain, hydroxyl and cyclo-fatty acids made up of C12: 0, C13:0, C14:0 iso, C14:0, C15:0 iso, C15:0 anteiso, C15:1 w 8c, C15:0, C16:0 iso, C16:1w5c, C16:0, C15:030H, C17:1 $\omega$ 8c, C17:0 cyclo, C17:0, C18:1 $\omega 5 \mathrm{c}$ and C18:0. $R$. rhodochrous was dominated by straight chain, monounsaturated and 10-methyl fatty acids. The inability to synthesize branched, cyclo- and hydroxyl- fatty acids, was observed in $R$. rhodochrous which composed mainly of C14: 0, C15: $1 \omega$ 5c, C15:0, C16:1 $\omega$ 9c, C16:0, C17:1 $\omega$ 8c, C17:0, C17:0 10-methyl, C18: $1 \omega$ 9c, C18.0, 10 methyl-C 18:0 TBSA, C20:1 $\omega$ 9c, and C20:0. Descriptive statistics reveal a mean of 2.53, 15.10 and $\mathbf{1 5 . 1 5}$ for retention time (RT), equivalent chain length (ECL) and Peak name, respectively. Possible implications of the variations in fatty acid distribution may include differences in their abilities to produce various secondary metabolites and potentials to degrade a variety of xenobiotics.
\end{abstract}

Keywords: Fatty acids, paints, Rhodococcus rhodochrous, Klebsiella pneumoniae Corresponding author email: oobidi@unilag.edu.ng

\section{Introduction}

An important component of cellular materials in many living organisms is the fatty acid, which also helps in identification and characterization of microbial community structure. Fatty acids are the basic building blocks of more complex lipids (Jenkins et al., 2015). Therefore, their composition in different bacterial species can be used as a means for comparisons. The qualitative nature of gas chromatographic data allows for the authentic microbial biomass composition differences in a diverse community. Essentially, by virtue of biosynthetic relationships, fatty acids can be categorized into two groups comprising the straight chain fatty acids (SCFAs) and the branched-chain fatty acids (BCFAs). Branchedchain fatty acids (isobutyric acid, 2methylbutyric acid, and isovaleric acid) are primarily saturated fatty acids, both even-carbonand odd-carbon-numbered, with one or more methyl branches, accordingly called mono- or multi-methyl BCFAs, respectively (Maheshwari et al., 2020). They are mainly derived from the catabolism of branched-chain amino acids such as valine, leucine, and isoleucine ( $\mathrm{He}$ et al., 
2019). SCFAs (formic acid, acetic acid, propionic acid, butyric acid, and valeric acid) are predominant end products of fermentation of dietary fibers by the anaerobic intestinal microbiota (He et al., 2019). Previous studies have shown that bacteria with the straight-chain membrane system usually need unsaturated fatty acids for their growth. On the contrary, these fatty acids are not necessarily required for growth by bacteria with the branched-chain membrane system such as the iso-, anteiso-, and $\omega$-alicyclic fatty acids (Cronan, 2006). Klebsiella pneumoniae, a human saprophyte in the nasopharynx and in the intestinal tract, has been shown to be the most medically important species of the genus Klebsiella known to cause community-acquired bacterial pneumonia, occurring particularly in chronic alcoholics (Cillóniz et al., 2019). Previous studies also show that Klebsiella, an ubiquitous pathogen (Struve and Krogfelt, 2004) second only to Escherichia coli accounts for $13.1 \%$ of nosocomial urinary tract infections (UTI) with especially higher occurrence in immunocompromised patients such as patients with neuropathic bladders or with diabetes mellitus (Fernando et al., 2017). Interestingly, $K$. pneumoniae has also been most commonly isolated from environmental and aquatic samples. Incidentally, the environmental isolates have been described as being indistinguishable from human clinical isolates with respect to their biochemical reactions and virulence (Podschun et al., 2001). The $K$. pneumoniae membrane is characterized by a fatty acid profile dominated to a large extent $(50 \%)$ by the straight chain fatty acids, with the major ones being $\mathrm{C} 12: 0, \mathrm{C} 13: 0, \mathrm{C} 14: 0, \mathrm{C} 15: 0$, $\mathrm{C} 17: 0, \mathrm{C} 18: 0$ and $\mathrm{C} 19: 0$ as well as -iso branched fatty acids and hydroxylated fatty acids (Vasyurenko et al., 2001). Members of the actinomycetes have been widely studied because of their ability to produce various secondary metabolites and extremely high potentials to degrade a variety of xenobiotics, including toxic aromatic compounds as pyridine (Yoon et al., 2000). Rhodococcus species have been found to contain C16:0, C18:1 cis9, 10-methyl-C-18:0 (TBSA) as major fatty acids (Yoon et al., 2000). In the present study, variations in fatty acid profiles were investigated using the MIDI Sherlock system to analyze model spoilage organisms previously isolated from paints (Obidi et al., 2017) based on pattern recognition software and the database of the Sherlock data file. The study is important because the composition of fatty acids in different bacterial species can be used as a means for comparison as well as predict spoilage potentials in paint microbes.

\section{Materials and Methods}

\section{Microorganisms}

Klebsiella pneumoniae and Rhodococcus rhodochrous were already isolated in our earlier work (Obidi et al., 2017). The isolates were maintained as stock cultures on Nutrient Agar (Difco) slants. Fresh ( $24 \mathrm{~h}$ ) cultures were each streaked on a 4-quadrant pattern on two tryptic soy agar (TSA) plates and incubated at $28^{\circ} \mathrm{C}$. They were harvested from the third quadrant with a sterile spatula into the bottom of a glass screw-cap test tube (MIDI, 2001).

\section{Extraction}

Extraction of the fatty acids from the bacterial cells was carried out using a reagent made up of $15 \% \mathrm{NaOH}$ in methanol and water (1:1). Subsequently, saponification was carried out by adding $0.1 \mathrm{ml}$ of reagent 1 (methanolic base) into the culture tubes and covered tightly. The tubes were then vortexed for 5-10 seconds in three successions and subsequently placed in a rack and incubated in a $100^{\circ} \mathrm{C}$ water bath for $5 \mathrm{~min}$, cooled briefly for about 60 secs and reincubated in the water bath for additional $25 \mathrm{~min}$. The methylation process which ensures the conversion of the sodium salts of the fatty acids to fatty acids methyl esters was carried out for 10 mins at $80^{\circ} \mathrm{C}$ following an initial vortexing for 510 secs. Two (2.0) $\mathrm{ml}$ of the methylation reagent (6 N HCl-methanol solution, 3.25:2.75) was added into the tubes and covered tightly. The tubes were subsequently cooled in a tray of cold water. The fatty acid methyl esters were extracted from the acidic aqueous phase and transferred to an organic phase with a liquidliquid extraction process with the addition of 1.0 $\mathrm{ml}$ of an extraction solvent (1:1 mixture of hexane and methyl tert-butyl alcohol). The tubes were tightly capped, placed in the hemocytometer and mixed end-over-end for 10 mins. Subsequently, a clean Pasteur pipette was used to remove and discard the aqueous (lower) phase as described by MIDI. Flame ionization 
detection gas chromatography was performed using the MIDI system (Newark, Del.) and the various peaks were established. Retention time data was obtained from injection of the calibration mixture and is converted to Equivalent Chain Length (ECL) data for naming of the bacterial fatty acids. The ECL value for each fatty acid was therefore derived as a function of its elution time in relation to the elution times of a known series of fatty acids. The individual fatty acids were characterized by a pattern recognition software based on comparison to commercially available databases (MIDI, Inc.). Descriptive statistics involving minimum, maximum, mean, standard deviation and variance were computed for RT, ECL and Peak Name.

\section{Results}

The fatty acids isolated from $K$. pneumoniae and $R$. rhodocochrous were characterized by gas chromatographic analysis and compared with the fatty acid profiles of many known bacterial species using pattern recognition software. The fatty acid chromatograms generated were studied and contrasted. Branched, hydroxyl and cyclo fatty acids were observed in $K$. pneumoniae (Table 1 ). Table 1 shows the identification of the various cellular fatty acids of $K$. pneumonia as well as the differences in retention time (RT) and equivalent chain length (ECL) of the various fatty acids observed. In $K$. pneumoniae RT ranged from 1.6302 to 3.4279 . In contrast, certain fatty acids observed in $K$. pneumoniae were not detected in $R$. rhodochrous, which instead possessed distinctive methyl fatty acids (Table 2). Table 2 displays the identification of types of cellular fatty acids of $R$. rhodochrous as well as differences in retention time (RT) and equivalent chain length (ECL) of the various fatty acids observed. In $R$. rhodochrous, RT ranged from 2.178 to 4.0321 . However, some similarities were observed between the two organisms. Straight chain fatty acids predominate $(41 \%$ and $46 \%)$ in each case and a good proportion ( $23.5 \%$ and $38 \%$ ) of monounsaturated fatty acids were detected in both organisms. $K$. pneumoniae produced seven major straight chain fatty acids (C12:0, C13:0, C14:0, C:15: 0, C16:0, C17:0 and C18:0), two branched chain fatty acids (anteisoC15 and iso-C16), one hydroxyl fatty acid $(\mathrm{C} 15: 03 \mathrm{OH})$, three monounsaturated fatty acids (C15:1 $\omega$ 8c, C16:1 $\omega$ 5c and C17:1 $\omega$ 8c) and one cyclo-fatty acid (C17:0 cyclo) (Table 1). $R$. rhodochrous, on the other hand, produced thirteen fatty acids comprising six straight chain fatty acids (C14:0, C15:0, C16:0, C17:0, C18:0 and $\mathrm{C20:0);} \mathrm{five} \mathrm{monounsaturated} \mathrm{fatty} \mathrm{acids}$

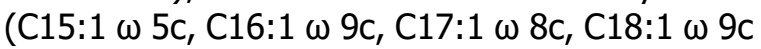
and $\mathrm{C} 20: 1 \omega \mathrm{cc}$ ) (Table 2), and in addition to these, $R$. rhodochrous was found to produce two methyl fatty acids (C17:0 10 methyl and C18:0 10 methyl, TBSA). Figures 1 and 2 show the gas chromatograms of the bacterial fatty acids isolated from the two organisms. The straight chain fatty acids (C:12:0, C13:0, C14:0, C15:0, $\mathrm{C} 16: 0, \mathrm{C} 17: 0, \mathrm{C} 18: 0, \mathrm{C} 19: 0$ and $\mathrm{C} 20: 0)$ were the most abundant fatty acids in both organisms. The monounsaturated fatty acids (C15:1 $\omega$ 5c, C15:1 $\omega 8 \mathrm{c}, \mathrm{C} 16: 1 \omega \mathrm{cc}, \mathrm{C} 17: 1 \omega \mathrm{cc}$, C18:1 $\omega$ 5c, C18:1 $\omega$ 9c and $\mathrm{C} 20: 1 \omega 9 \mathrm{c})$ generally, were next in order of abundance. The hydroxylated fatty acids $(\mathrm{C} 15: 03 \mathrm{OH})$ and cyclo propane fatty acids (C17:0 cyclo) were of low abundance and occurred only in $K$. pneumoiae. The results of the study show obvious differences in the fatty acid distribution patterns of the two organisms investigated. An obvious inability to synthesize the cyclo, hydroxyl and branched fatty acid was seen in $R$. rhodochrous. Klebsiella pneumoniae had $41 \%$ of straight chain fatty acids compared to $R$. rhodochrous which had $46 \%$. $K$. pneumoniae was observed to possess $23.5 \%$ of monounsaturated fatty acids, $6 \%$ of hydroxyl fatty acids, $6 \%$ of cyclo- fatty acids and $23.5 \%$ of branched- chain fatty acids. $R$. rhodochrous on the other hand had $38 \%$ of monounsaturated fatty acids and $15.3 \%$ methyl fatty acids. The differences in fatty acid profiles thus, constitutes the major distinguishing features of $R$. rhodochrous and $K$. pneumoniae in addition to some established distinctiveness in their phenotypic, phylogenetic and genetic characteristics.

Figures 3 and 4 show each variable in the dataset. The upper tail indicates the maximum value, while the lower tail represents the minimum value. The middle line across the boxplot represents the median ( $2^{\text {nd }}$ quartile) of each variable in the dataset. For $K$. pneumoniae, the mean is 2.53, 15.10, 15.15; SD is $0.45,1.50$ and 1.47; while variance is $0.206,2.251$ and 2.163 for RT, ECL and Peak Name respectively. In the case of $R$. rhodochrous, mean is 3.03, 16.74, 16.8; SD is $0.52,1.67,1.67$ while variance is $0.269,2.774$ and 2.816 for RT, ECL and Peak name 
Obidi, O.F. Nig. J. Biotech. Vol. 37 Num. 2: 47-55 (Dec 2020)

respectively. The result shows that there is a slight difference between the two major variables
( $K$. pneumoniae, $R$. rhodochrous) based on the number of observations analyzed.

Table 1: Identification of Cellular Fatty Acids of K. pneumoniae

\begin{tabular}{llll}
\hline RT & ECL & Peak Name & Percent \\
\hline 1.6302 & 12.0004 & $12: 0$ & 0.42 \\
1.8917 & 12.9999 & $13: 0$ & 0.16 \\
2.0733 & 13.6288 & $14: 0$ iso & 0.12 \\
2.1806 & 13.9998 & $14: 0$ & 12.24 \\
2.3730 & 14.6309 & $15: 0$ iso & 0.06 \\
2.4014 & 14.7242 & $15: 0$ anteiso & 0.45 \\
2.4296 & 14.8168 & $15: 1 \omega 8 \mathrm{c}$ & 0.08 \\
2.4859 & 15.0009 & $15: 0$ & - \\
2.6843 & 15.6321 & $16: 0$ iso & 0.17 \\
2.7760 & 15.9240 & $16: 1 \omega 5 \mathrm{c}$ & 0.29 \\
2.8009 & 16.0033 & $16: 0$ & 31.85 \\
2.9687 & 16.5358 & $15: 030 \mathrm{H}$ & 0.08 \\
3.0567 & 16.8149 & $17: 1 \omega \mathrm{cc}$ & 0.38 \\
3.0875 & 16.9127 & $17: 0$ cyclo & 1.14 \\
3.1149 & 16.9998 & $17: 0$ & 0.40 \\
3.4068 & 17.9319 & $18: 1 \omega 5 \mathrm{c}$ & 0.07 \\
3.4279 & 17.9994 & $18: 0$ & 0.17
\end{tabular}

$\mathrm{RT}$, retention time; $\mathrm{ECL}$, equivalent chain length

Table 2: Identification of Cellular Fatty Acids of $R$. rhodochrous

\begin{tabular}{llll}
\hline RT & ECL & Fatty Acid & Percent \\
\hline 2.1780 & 13.9999 & $14: 0$ & 1.46 \\
2.4589 & 14.9217 & $15: 1 \omega 5 c$ & 0.36 \\
2.4833 & 15.0017 & $15: 0$ & \\
2.7339 & 15.7989 & $16: 1 \omega 9 c$ & -- \\
2.7971 & 15.9998 & $16: 0$ & 0.43 \\
3.0543 & 16.8155 & $17: 1 \omega 8 c$ & 22.13 \\
3.1128 & 17.0013 & $17: 0$ & 7.11 \\
3.2419 & 17.4130 & $17: 010-$ methyl & 2.93 \\
3.3606 & 17.7918 & $18: 1 \omega 9 c$ & 1.16 \\
3.4255 & 17.9988 & $18: 0$ & 32.83 \\
3.5465 & 18.3944 & $18: 010-$ methyl, TBSA & 1.62 \\
3.9684 & 19.7884 & $20: 1 \omega 9 c$ & 6.13 \\
4.0321 & 20.0002 & $20: 0$ & 0.63 \\
& & & 0.35
\end{tabular}

$\mathrm{RT}$, retention time; $\mathrm{ECL}$, equivalent chain length 
Obidi, O.F. Nig. J. Biotech. Vol. 37 Num. 2: 47-55 (Dec 2020)

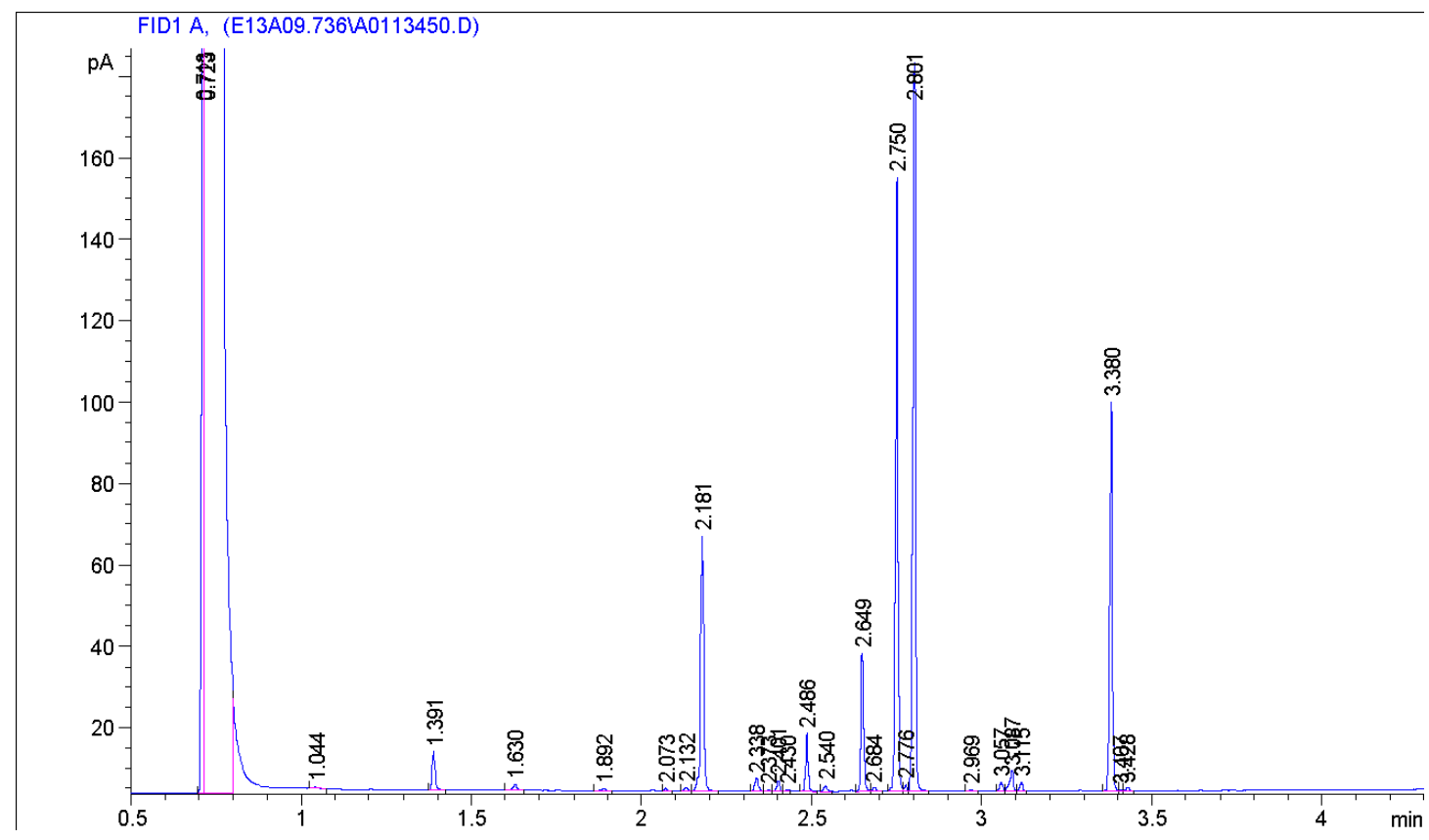

Fig. 1: Chromatogram of fatty acids of Klebsiella pneumoniae



Fig. 2: Chromatogram of fatty acids of Rhodococcus rhodochrous 




Fig. 3: Box Plot of $K$. pneumoniae Chromatogram parameters

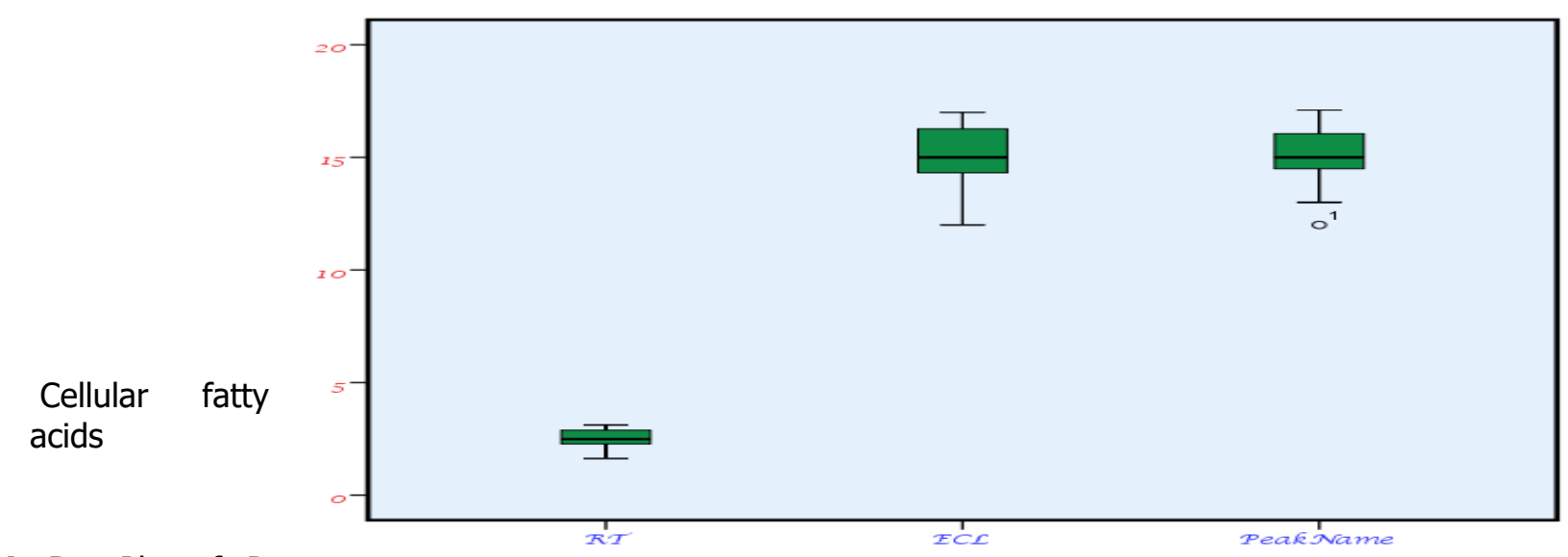

Fig. 4: Box Plot of $R$. rhodochrous Chromatogram parameters

\section{Discussion}

In the present study, fatty acid methyl ester (FAME) analysis was used to evaluate and compare the fatty acid profiles of $K$. pneumoniae and $R$. rhodococcus. The disparities in the fatty acid composition found in $K$. pneumoniae and $R$. rhodochrous are not unusual, being physiologically, biochemically, metabolically and taxonomically different organisms. Klebsiella pneumoniae was observed to possess $41 \%$ of straight chain fatty acids and $11.8 \%$ branched fatty acids compared to $R$. rhodochrous which had $46 \%$ of straight chain fatty acids and no branched fatty acids. This is in agreement with a previous report by Lingfa et al., (2020) which shows that straight chain fatty acids occur most commonly in bacteria and usually constitute about $10 \%$ of the total cellular fatty acids in bacteria which contain mostly branched-chain fatty acids. All the saturated fatty acids, including the branched chain ones, are synthesized from acetyl coenzyme $A$ (acetyl-CoA) as the primer and malonyl-CoA as the chain extender, followed, in some cases, by a modification of the fatty acid products. It is therefore believed that the enzyme system of the organism in question is responsible for these differences. Although branched chain fatty acids and straight chain fatty acids both increase membrane fluidity, they do not yield cells with identical morphologies or fitness for tolerating cold stress. Fatty acids having chain lengths shorter than 14 carbon atoms were observed in $K$. pneumoniae in contrast to $R$. rodochrous. This is in reasonable agreement with the report of (Wu et al., 2020) who observed shorter chain lengths of fatty acids in $K$. pneumonia compared to other isolates. A sensible reason may be that the cofactors involved in the synthesis are different and favor 
the synthesis of shorter chain fatty acids in $K$. pneumoniae. Generally, the fatty acid compositions of bacteria are usually different from those of higher organisms in the sense that they have no polyunsaturated fatty acids (fatty acids with more than one unsaturation) (Tan et al., 2012; Torres et al., 2014; Bazinet and Laye, 2014). Substantial amounts (38\%) of unsaturated and branched chain fatty acids were observed in $R$. rhodochrous. This might also contribute to having a cell envelope with greater flexibility. Similar results were obtained by Yoon et al., (2000) who reported the occurrence of saturated, unsaturated and 10-methyl-branched fatty acids (10-methyl C18:0 TBSA) and major amounts of $\mathrm{C} 16: 0, \mathrm{C} 18: 0$ in several species of Rhodococcus tested. Branched chain fatty acids also have been found to enhance growth and virulence in bacteria and might give a cell envelope greater flexibility (Kaiser et al., 2016). Ante-iso and iso branching (that is on the antepenultimate and penultimate carbons) have been reported to modify the thermotropic behavior and enhance the fluidity of lipid bilayers. Previous studies found that methyl branching reduces lipid condensation, decreases the bilayer thickness, and lowers chain ordering (Poger et al., 2014). In addition, branching also results in the formation of kinks at the branching point, thereby enhancing the fluidity of lipid bilayers (Poger et al., 2014). In this study, $K$. pneumoniae, an important pathogen, had branched chain fatty acids. Previous study has demonstrated the connection between pathogenicity and possession of branched-chain amino acids which are precursors for branched chain fatty acids (Kaiser et al., 2018). Branchedchain amino acids represent important nutrients in bacterial physiology. They do not only support proliferation during infection, but also the evasion of host defenses (Kaiser and Heinrichs, 2018). The branched-chain amino acids are vital to both growth and virulence (Kaiser et al., 2016). This probably explains why Klebsiella is highly virulent and highly pathogenic. The predominance of straight chain fatty acids in both organisms have been documented (Yoon et al., 2000). So far, the branching of fatty acids have been described as being widespread in prokaryotic membranes. These branched-chain fatty acids are however, less common than the straight-chain fatty acids (Poger et al., 2014). The fatty acid profile observed in the $K$. pneumoniae is similar to those previously reported (Ringelberg et al., 2008). An interesting occurrence of cyclo and hydroxylated fatty acids is particularly notable. The hydroxylated fatty acids $(\mathrm{C} 15: 03 \mathrm{OH})$ and cyclo propane fatty acids (C17:0 cyclo) were of low abundance and occurred only in $K$. pneumoiae. This corroborates the report of Sohlenkamp and Gieger, (2016) who reported the occurrence of 3-Hydroxy straight-chain fatty acids in Gram-negative bacteria as the major acyl component of lipopolysaccharides in cell walls. Some researchers have indicated that a higher amount of Cyclo 17:0 indicates probable increase in certain groups of Gram-negative bacteria (Ibekwe and Kennedy, 1999). To further corroborate this fact, Ringelberg et al., (2008) reported that $n 16: 1 w 7 c, n 18: 1 w 9 c$ and $n 18: 1 w 7 c$ are bio-markers of fatty acids for Gram-negative bacteria. The reduced proportion of monounsaturated fatty acids $(23.5 \%)$ in $K$. pneumoniae is usual among Gram-negative organisms. $R$. rhodococcus has been shown to possess physiological properties that are distinguishable from those of $K$. pneumoniae. It was observed by Yoon et al., (2000) that in particular, the ability of Rhodococcus species to degrade pyridine was uncommon. Meanwhile, the ability to degrade pyridine has not been reported in $K$. pneumoniae. However, $K$. pneumoniae has been observed to tolerate and degrade tannic acid in very high concentrations. It is noteworthy that tannins, which serve as immune compounds in plants and protect them from ruminant and microbial attacks (Huang et al., 2018) due to their toxicity due to microbial enzyme inhibition and substrate deprivation action on microbial membranes, were degraded by $K$. pneumoniae. The adaptation of $K$. pneumoniae to the toxicity of tannic acids has been linked to an increase in the degree of saturation of membrane lipid fatty acids (Pepi et al., 2013).

The occurrence and prevalence of unsaturated fatty acids which are common to both organisms have been reported to play only a minor role in stress adaptation (de Carvalho and Caramujo, 2018) and are agents specific to aerobic bacteria (Erdogan et al., 2013), and therefore may not have contributed to the degradation potentials observed in both organisms.

\section{Conclusion}


The study clearly showed that a fatty acid pattern consisting of a large proportion of straight chain, branched chain, hydroxyl fatty acids and a substantial amount of cyclo fatty acids is characteristic of $K$. pneumoniae (a typical Gramnegative organism) and may contribute to its degradative and physiological potentials. The absence of cyclo, hydroxyl and branched fatty acids was also confirmed to be typical in Rhodococcus species.

\section{Acknowledgement}

The author is grateful to the American Association of University Women (AAUW) for an international postdoctoral grant to support this research as well as Professor Larry J Halverson for useful discussions and technical assistance.

\section{References}

Bazinet, R. P.and Layé, S. (2014). Polyunsaturated fatty acids and their metabolites in brain function and disease. Nat Rev Neuroscience, doi:10.1038/nrn3820.

Cillóniz, C., Dominedò , C. and Torres, A. (2019). Multidrug Resistant Gram-Negative Bacteria in Community-Acquired Pneumonia. Critical Care 23(79): https://doi.org/10.1186/s13054-0192371-3

Cronan, J. E. (2006). A bacterium that has three pathways to regulate membrane lipid fluidity. Molecular Microbiology 60(2): 256-259. doi.org/10.1111/j.1365-2958.2006.05107.x Duggan, J. M., Oldfield, G. S. and Ghosh, H. K. (1985).Septicaemia as a hospital hazard. J. Hosp. Inf. 6:406-412.

de Carvalho, C.C.C.R. and Caramujo, M. J. (2018). The Various Roles of Fatty Acids Molecules, doi: $10.3390 /$ molecules 23102583

Erdoğan, E. E., Şahin, F. and Naml, A. (2013). Phospholipid fatty acids analysis-fatty acid methyl ester (PLFA-FAME) changes during bioremediation of crude oil contamination soil. Afr. J. Biotechnol. 12(44): 6294-6301.
Fernando, M.M.P.S., Luke, W.A.N.V., Mithithinda, J.K.N.D., Wickramasinghe, R.D.S.S., Sebastiampillai, B.S., Gunathilake, M.P.M.L., Silva, F.H.D.S. and Premaratna, R. (2017). Extended spectrum beta lactamase producing organisms causing urinary tract infections in Sri Lanka and their antibiotic susceptibility pattern A hospital based cross sectional study. BMC Infectious Diseases, 17, 138 (2017). https://doi.org/10.1186/s12879-017-2250-y

Huang, Q., Liu, X., Zhao,G., Hu, T. and Wang, Y (2018). Potential and challenges of tannins as an alternative to in-feed antibiotics for farm animal production. Anim Nutr. 2018, 4(2): 137-150.

He, L., Prodhan, A. I., Yuan, F., Yin, X., Lorkiewicz, P. K., Wei, X., Feng, W., McClain, C. and Zhang, X. (2018). Simultaneous Quantification of Straight-chain and Branchedchain Short Chain Fatty Acids by Gas Chromatography Mass Spectrometry, J Chromatogr B Analyt Technol Biomed Life Sci. 1092: 359-367. doi:10.1016/ j.jchromb.2018.06.028.

Ibekwe, A. M. and Kennedy, A. C. (1999). Fatty acid methyl ester (FAME) profiles as a tool to investigate community structure of two agricultural soils. Plant and Soil. 206: 151-161.

Jenkins, B., West, J. A. and Koulman A. A. (2015). Review of odd-chain fatty acid metabolism and the role of pentadecanoic acid (C15:0) and heptadecanoic acid (C17:0) in health and disease. Molecules, 20; 2425-2444. Doi: 10.3390/Molecules 20022425

Lingfa, L., Nathiya T.C.H., Prudhvi, V.K., Sharmila, R., Deepa, R. and Rajan, A. P. (2015). Study of membrane fatty acids of Gram negative bacteria and its influence towards the terrestrial ecosystem. International Journal of pharmaceutical, Biological and Chemical Sciences, 4(2): 1-19

Kaiser, J. C., Sen, S., Sinha, A., Wilkinson, B. J. and Heinrichs, D. E. (2016). The role of two branched-chain amino acid transporters in Staphylococcus aureus growth, membrane fatty acid composition and virulence. Mol Microbiol, 70:586-599. doi: 10.1111/mmi. 
Kaiser,J. C., Heinrichs, D. E. (2018). Branching Out: Alterations in Bacterial Physiology and Virulence Due to Branched-Chain Amino Acid Deprivation. MBio. 9(5): 1-17.e01188-18

Maheshwari, G., Ringseis ,R. , Wen, G., Gessner, D.K., Rost, J., Fraatz, M. A., Zorn, H. and Eder,K. (2020). Branched-Chain Fatty Acids as Mediators of the Activation of Hepatic Peroxisome Proliferator-Activated Receptor Alpha by a Fungal Lipid Extract. Biomolecules, 10, 1259; doi:10.3390/biom10091259

MIDI, Inc. Sherlock Microbial Identification System References (2001).

Obidi, O. F., Wenner, S. and Halverson, L. (2017). Molecular based analysis of bacterial community structure of spoilt latex paints in Nigeria. Egyptian J Exp Biol. 13(2):145-149.

Pepi, M., Cappelli, S., Hachicho, N., Perra, G., Renzi, M., Tarabelli, A. (2013). et al. Klebsiella sp. strain C2A isolated from olive oil mill waste is able to tolerate and degrade tannic acid in very high concentrations. FEMS Microbiol Lett. 343: 105112

Podschun, R., Pietsch, S. Höller, C. and Ullmann, U. (2001). Incidence of Klebsiella Species in Surface Waters and Their Expression of Virulence Factors. Appl and Env. Micobiol. 87(7): 33253327.

Poger, D., Caron, B. and Mark, A. E. (2014). Effect of methyl branched fatty acids on the structure of lipid bilayers. J Phy Chem B. 118 (48): 13838-13848. DOI: $10.1021 /$ jp503910r.

Ringelberg, D., Richmond, M., Foley, K. and Reynolds, C. (2008). Utility of lipid biomarkers in support of bioremediation efforts at army sites. $J$. Microbiol Met. 74(1):17-25.

Sohlenkamp, C. and Geiger, O. (2016). Bacterial membrane lipids: diversity in structures and pathways. FEMS Microbiology Reviews, 40 (1), 133-

159, https://doi.org/10.1093/femsre/fuv008

Struve, C. and Krogfelt, K. A. (2004). Pathogenic potential of environmental Klebsiella pneumoniae isolates. Environmental Microbiology
(2004) 6(6), 584-590. doi:10.1111/j.14622920.2004.00590.x

Tan, Z. S., Harris, W. S., Beiser, A. S., Au, R., Himali, J. J., Debette, S. (2012). et al. Red blood cell omega-3 fatty acid levels and markers of accelerated brain aging. Neurol. 2012; 78, 658664.

Torres, M., Price, S. L, Fiol-deRoque, M. A., Marcilla-Etxenike, A., Ahyayauch, $\mathrm{H}$,

Barceló-Coblijn G. et al. (2014). Membrane lipid modifications and therapeutic effects mediated by hydroxydocosahexaenoic acid on Alzheimer's disease. Biochimica Biophysica Acta (BBA) Biomembranes 1838: 1680-1692.

Vasyurenko, Z. P., Opanasenko, L. S., Koval', G.M., Turyanitsa, A. I., Ruban, N. M. (2001). Cellular and lipopolysaccharide fatty acid composition of the type strains of Klebsiella pneumoniae, Klebsiella oxytoca, and Klebsiella nonpathogenic species. Mikrobiology, 63(3):1321.

Wu, T., Xu, F., Su,C., Li, H., Na Lv', Liu,Y., Gao,Y., Lan'Y. and Li, J.(2020). Alterations in the gut microbiome and cecal metabolome during Klebsiella pneumoniae-Induced Pneumosepsis. Frontiers in Immunology 11(1331):1-13. doi: 10.3389/fimmu.2020.01331

Yoon, J. H., Kang, S.S., Cho, Y.G., Lee, S. T. K., Yung, H., Kim, C. J. et al. (2000). Rhodococcus pyridinivorans sp. nov., a pyridine-degrading bacterium. International J. Syst Evol. Microbiol. 50, 2173-2180. 